\title{
Using Near Detector(s) to predict the Far Detector Events in NOvA Experiment
}

\author{
Zelimir Djurcic \\ Argonne National Laboratory, 9700 South Cass Avenue, Argonne, IL 60439, USA
}

\begin{abstract}
The NOvA experiment is designed to search for a non-vanishing mixing angle $\theta_{13}$ with unprecedented sensitivity and has the potential to resolve the neutrino mass hierarchy and constrain CP-violation phase. NOvA will use two functionally identical detectors at near and far locations to eliminate sensitivity to modeling of neutrino flux and cross-sections. The near detector will measure neutrino rate to constrain backgrounds expected in the far detector which will search for appearance of electron neutrinos and/or anti-neutrinos using Fermilab NuMI neutrino beam. This report describes initial thoughts on how the available beams and detectors may be used to reach the NOvA goals.
\end{abstract}

Keywords: Neutrino Interactions, Neutrino Oscillations

PACS: $13.15 .+\mathrm{g}, 14.60 . \mathrm{Pq}$

\section{INTRODUCTION}

Recent years have seen enormous progress in the physics of neutrino mixing, but several critical questions remain: What is the value of $\theta_{13}$, the last unmeasured mixing angle in the neutrino mixing matrix? What is the mass hierarchy? Do neutrino oscillations violate CP symmetry? Why are the quark and neutrino mixing matrices so different? The value of $\theta_{13}$ is central to each of these questions and sets the scale for experiments needed to resolve the mass hierarchy and to search for CP violation. In addition, the size of $\theta_{13}$ with respect to the other mixing angles may give insights into the origin of these angles and the source of neutrino mass. While both accelerator and reactor experiments hold the promise of unambiguously determining the $\theta_{13}$ mixing angle, the NOvA (NuMI Off-Axis $v_{e}$ Appearance Experiment) is an accelerator based experiment that will help address all of the questions listed above [1]. The measurement of $\theta_{13}$ at NOvA will be conducted by searching for an appearance of electron neutrinos and/or anti-neutrinos in the NuMI (Neutrinos at the Main Injector) neutrino beam at Fermilab. Neutrinos for NOvA will be produced by $120 \mathrm{GeV}$ protons incident on a graphite target. The Fermilab accelerator complex is currently capable of delivering 400 kilowatts of power to the NuMI beam, but part of the NOvA project will include an upgrade to the accelerator to provide 700 kilowatts of power to NuMI. The experiment will use two functionally identical detectors: a 222 ton "Near" detector at a distance of about a 1000 $\mathrm{m}$ from the NuMI beam target and a large 15 kilo-ton "Far" detector at a distance of $810 \mathrm{~km}$ located in Ash River, Minnesota. The detectors will be located at an offaxis angle of $0.8^{0}$ with respect to the NuMI beam axis resulting in the neutrino flux confined to a relatively nar- row band of energy around $2 \mathrm{GeV}$, which is useful in limiting backgrounds in $v_{\mu} \rightarrow v_{e}$ searches. The detectors have a cell structure made of highly reflective extruded plastic PVC filled with liquid scintillator. The Far detector cells are $3.9 \mathrm{~cm}$ wide, $6.0 \mathrm{~cm}$ deep and $15.5 \mathrm{me}$ ters long. The Near detector cells are shorter with the same width and depth. Neutrinos interacting in the liquid produce charged particles with energy collected with wavelength-shifting fibers connected to photo-detectors. Such a scheme allows a very precise measurement of neutrino energy and an efficient separation of $v_{e}$ from $v_{\mu}$. The construction of the detectors is underway and the first neutrino detection has already achieved with the Near detector prototype located in a surface building at Fermilab. For the final location of the Near detector, a tunnel at the NuMI beam $0.8^{0}$ off-axis location will be excavated. The use of two detectors eliminates sensitivity to modeling of the neutrino flux and cross-sections, and only requires a knowledge of the relative acceptance of the two detectors. The cancellation of these important systematic errors will allow NOvA to explore the range of $\sin ^{2} 2 \theta_{13}$ at the 0.01 level. In this report we describe neutrino beams at hand and initial thoughts on how we plan to use these beams with available detectors to reach the NOvA goals.

\section{EXPECTED FLUXES AND EVENTS}

The un-oscillated $v$ spectrum expected at the NOvA Far detector from NuMI beam in neutrino mode (i.e. forward horn current configuration) is shown in Fig. 1. The flux is separated into contributions from pion and kaon parents. Pions produce neutrinos with average energies of about $2 \mathrm{GeV}$ while kaons have a much wider energy distribu- 
tion. Fig. 2 shows the same flux, now broken down into contributions from four neutrino types. It is now conve-

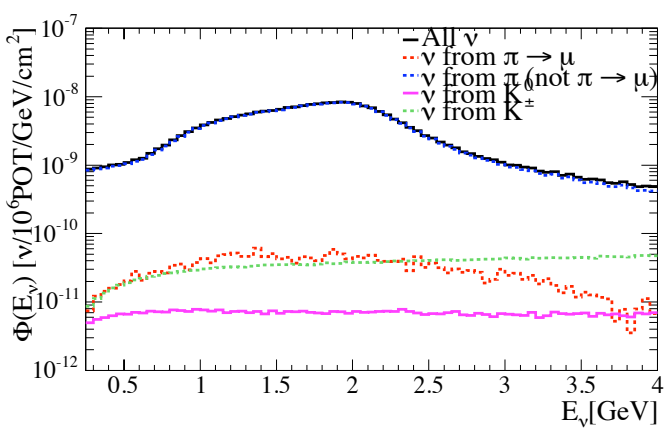

FIGURE 1. Flux expected at the NOvA Far detector in neutrino mode, broken down into contributions from $v$ parents.

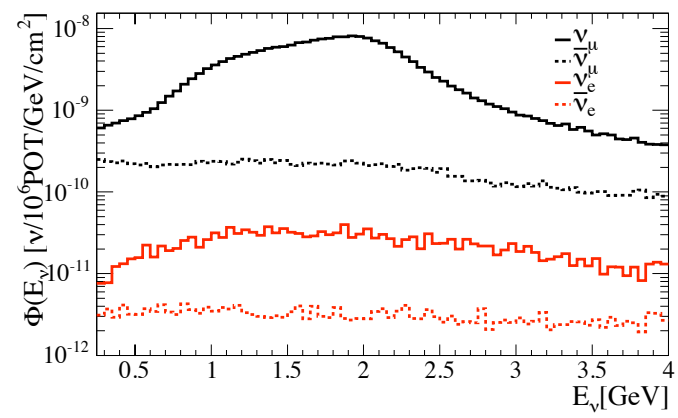

FIGURE 2. Flux expected at the NOvA Far detector in neutrino mode, broken down into four $v$ types.

nient to examine the neutrino spectrum expected at the Near detector: Figures 3 and 4 show expected fluxes broken down into contributions from neutrino parents and neutrino types, respectively. Comparing the Near and Far detector fluxes one finds them essentially identical, up to the differences attributed to different angular distributions of neutrinos with respect to the beam axis when seen at Near and Far detectors. A crucial component of

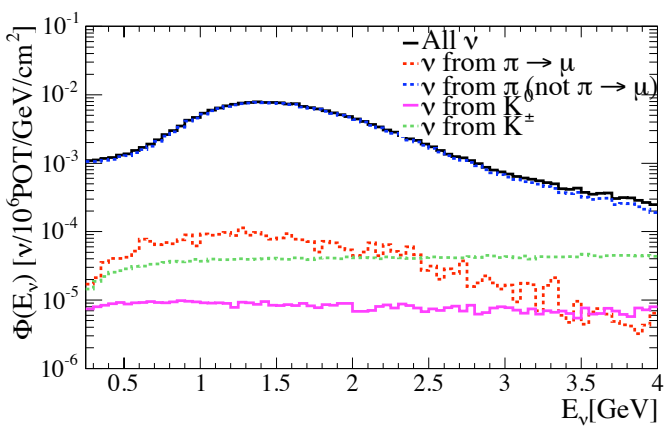

FIGURE 3. Flux expected at the NOvA Near Detector in neutrino mode, broken down into contributions from $v$ parents.

the NOvA experimental program is operation of the Near

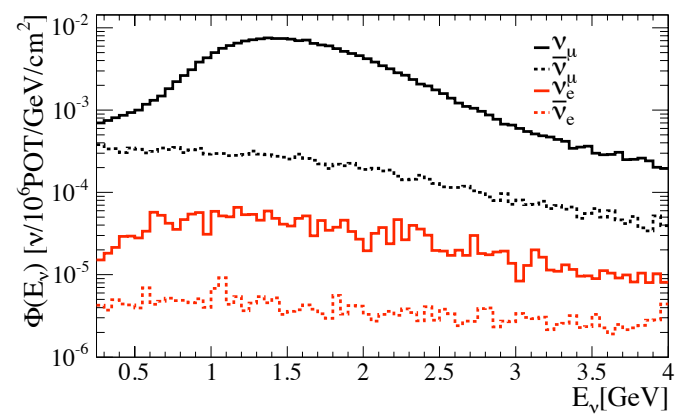

FIGURE 4. Flux expected at the NOvA Near Detector in neutrino mode, broken down into four $v$ types.

Detector On Surface (NDOS). When fully commissioned NDOS will be identical to the Near detector. While the final location of the Near detector will be underground at $0.8^{0}$ off-axis angle, NDOS is located on surface at an off-axis angle of about $6^{0}$. Figures 5 and 6 show expected fluxes broken down into contributions from neutrino parents and neutrino types, respectively. The kaon contribution to the flux is pronounced with the peak structure at $2 \mathrm{GeV}$ as expected at the given off-axis angle of $6^{0}$. Table 1 shows expected event rates at NDOS

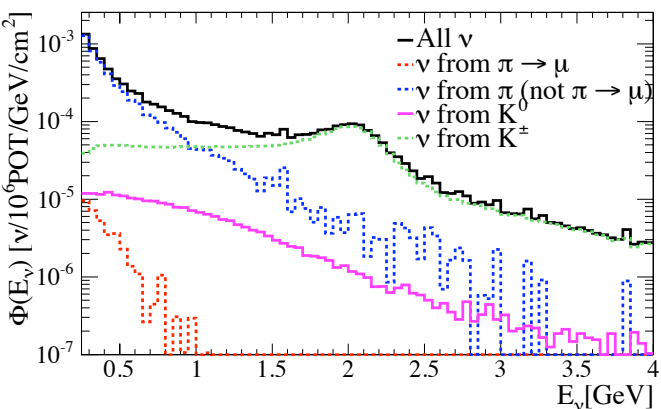

FIGURE 5. Flux expected at the NOvA Near Detector on surface (NDOS) in neutrino mode, broken down into contributions from $v$ parents.

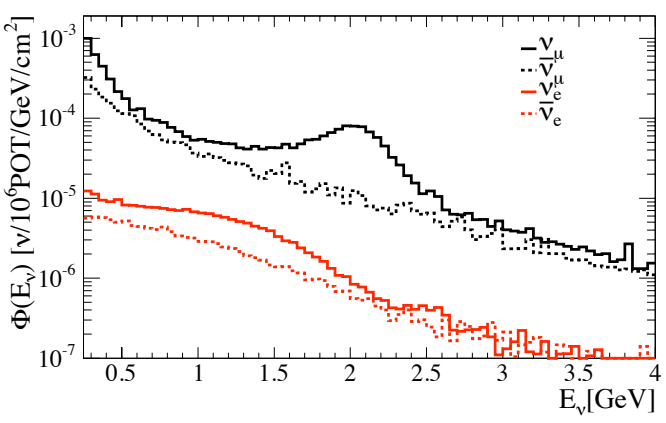

FIGURE 6. Flux expected at the NOvA NDOS detector in neutrino mode, broken down into four $v$ types. 
TABLE 1. Expected raw event rates at NDOS with $2.1 \times 10^{20}$ POT exposure from NuMI beam in neutrino mode.

\begin{tabular}{lllllll}
\hline $\mathbf{E}_{v}[\mathbf{G e V}]$ & $\begin{array}{l}\text { Total } \\
\mathbf{C C}\end{array}$ & $\begin{array}{l}\mathbf{C C} \\
\mathbf{Q E}\end{array}$ & $\begin{array}{l}\mathbf{C C} \\
\text { Res }\end{array}$ & $\begin{array}{l}\mathbf{C C} \\
\mathbf{D I S}\end{array}$ & $\begin{array}{l}\mathbf{C C} \\
\mathbf{C o h}\end{array}$ & $\mathbf{N C}$ \\
\hline$v_{\mu}$ Total & 4751 & 2288 & 1533 & 861 & 38 & 1911 \\
$v_{\mu} 1.6-2.4$ & 1931 & 559 & 842 & 511 & 20 & 699 \\
\hline$v_{e}$ Total & 340 & 166 & 119 & 50 & & 125 \\
\hline $\bar{v}_{\mu}$ Total & 624 & 323 & 179 & 103 & 14 & 353 \\
$\bar{v}_{\mu} 1.6-2.4$ & 132 & 50 & 55 & 24 & & 142 \\
\hline$v_{e}$ Total & 37 & 19 & 12 & 5 & & 19 \\
\hline
\end{tabular}

with $2.1 \times 10^{20}$ POT exposure from NuMI beam in neutrino mode, broken down into four neutrino types with charged current (CC) and neutral current (NC) contributions indicated. In addition, NDOS will detect neutrino interactions from the Booster beam currently used by the MiniBooNE experiment [3]. The unique capability of the NDOS program is the measurement of the NuMI off-axis beam at the large off-axis angle where the kaon components of the beam dominates, resulting in an enhanced intrinsic $v_{e}$ component. In fact, NDOS is located nearby the MiniBooNE detector at Fermilab where the NuMI off-axis beam has already been measured [2]. To validate the NuMI flux prediction we compared MiniBooNE data measured with NuMI neutrinos to the prediction for MiniBooNE based on beam Monte Carlo used by NOvA. The first result of the analysis is already published [2]. Here we repeated the analysis with an updated beam Monte Carlo. The result is shown in Fig 7. It shows

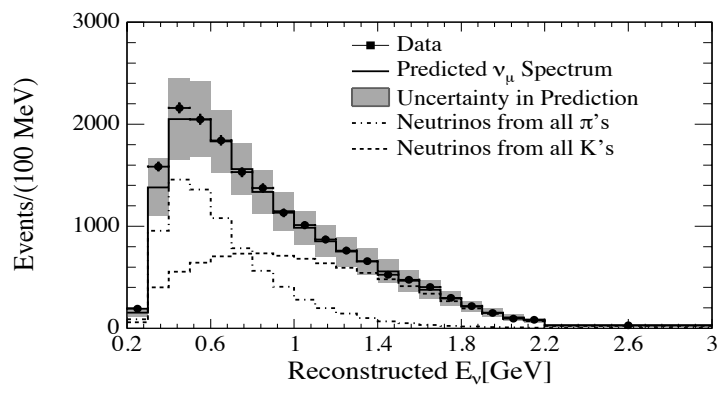

FIGURE 7. Reconstructed $E_{v}$ distribution of the NuMI offaxis $v_{\mu}$ CCQE candidate events in MiniBooNE.

that accurate predictions for the off-axis beam can be made. Therefore the Near detectors at the underground location and on the surface will provide constraints on both pion and kaon contributions to the beam, respectively. Given the identical functionality of the Near detector, NDOS and Far detectors the result will be an accurate prediction of event rates at the Far detector needed for the NOvA oscillation program. Expected raw signal and background rates at the Far detector are shown in Fig. 8. The NOvA detectors will be able to reject the $v_{\mu} \mathrm{CC}$ and
$\mathrm{NC}$ events at a level sufficient to make the hashed signal structure detectable. Space constraints in this report

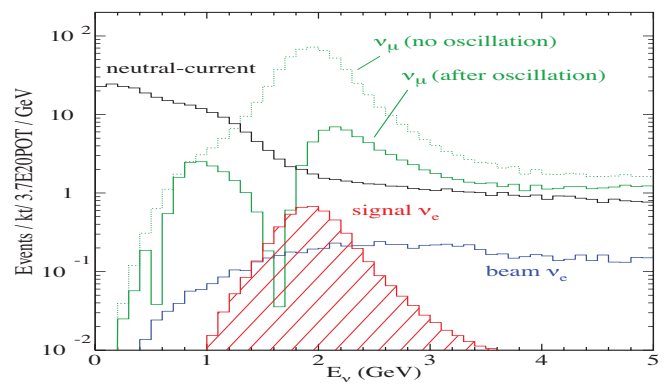

FIGURE 8. Expected raw signal and background rates at NO $v$ A Far detector. $v_{\mu}$ rates are shown with (green dotted) and without (green solid) oscillations applied. The NC rates are shown as a function of the visible energy and therefore pile up at low energies. The blue curve shows the intrinsic beam $v_{e}$ component. The red area is a signal at the $\mathrm{CHOOZ}$ limit.

only allowed detailed discussion of neutrino mode (forward horn current) fluxes and event rates, but the same approach can be followed when anti-neutrino events are analyzed. The expected sensitivity of the NOvA experiment to $v_{\mu} \rightarrow v_{e}$ transition expressed as a combination of $\theta_{13}$ and $\theta_{23}$ mixing angles with respect to $\delta_{C P}$ is shown in Fig. 9, assuming a conservative 10\% systematic error on the backgrounds, and 6 years of running evenly split between neutrino and anti-neutrino horn polarities.

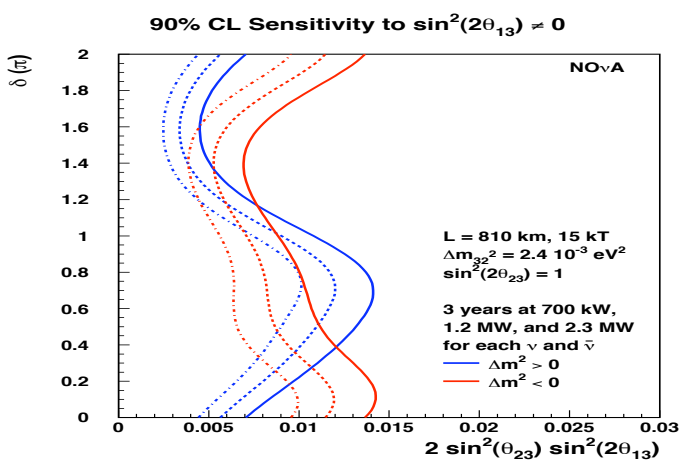

FIGURE 9. $90 \%$ C.L. sensitivity of the NOvA experiment to $v_{\mu} \rightarrow v_{e}$ oscillations. The blue curves assume normal mass hierarchy while the red curves show the inverted hierarchy case.

\section{REFERENCES}

1. D. Ayres et al., FERMILAB-DESIGN-2007-01.

2. P. Adamson et al., Phys. Rev. Lett. 102, 211801 (2009), arXiv:0809.2447 [hep-ex]; Z. Djurcic, "MiniBooNE Oscillation Results," arXiv:0907.3747 [hep-ex].

3. Z. Djurcic, "New Observations in MiniBooNE Experiment", these proceedings; A. A. Aguilar-Arevalo et al., Phys. Rev. Lett. 105, 181801 (2010). 